\title{
On the conjugate graphs of finite $p$-groups
}

\author{
Athirah Zulkarnain*, Nor Haniza Sarmin, Alia Husna Mohd Noor \\ Department of Mathematical Sciences, Faculty of Science, Universiti Teknologi Malaysia, 81310 UTM Johor Bahru, Johor, Malaysia \\ *Corresponding author: athirah.zulkarnain91@gmail.com
}

Article history

Received 13 February 2017

Accepted 21 March 2017

\begin{abstract}
Graphs can be related to groups by looking at its vertices and edges. The vertices are comprised of the elements or sets from the groups and the edges are the properties and conditions for the graph. Recently, researches on graphs related with groups have attracted many authors. A conjugate graph of a group is defined as; its vertex set is the set of non-central classes of $G$, and two distinct vertices $A$ and $B$ are connected by an edge if and only if they are conjugate. In this research, the conjugacy class of some finite $p$-groups are first found. Then, the conjugate graphs are determined.
\end{abstract}

Keywords: $p$-groups, conjugacy class, conjugate graph

\section{INTRODUCTION}

Studies on graphs of groups have been done extensively for the past few years. Also, many researches have been done on $p$-groups $(p$ is prime) as in [1-3]. In addition, varieties of graphs have been introduced by researchers such as conjugate graph which was introduced by Erfanian and Tolue [4].

Bertram et al. [5] studied the relation between the graph and the conjugacy classes of groups. In 2001, Segev [6] explored the relation between finite minimal nonsolvable groups with the commuting graph. The result showed that the commuting graph has diameter $\geq 3$.

In 2006, Akbari et al. [7] determined the diameter of commuting graph of the group and some of its subgroup. The results showed that if the graph is commuting graph, then the diameter is at most six for every field. In the same year, Bundy [8] studied the relation between commuting graph and its connectivity.

In 2008, Darafsheh [9] studied the relations between the noncommuting graphs with non-abelian group. He showed that when the non-commuting graph of a non-abelian group $G$ is isomorphic with the non-commuting graph of a non-abelian group $H$, this implies that the non-abelian group $G$ is isomorphic with the non-abelian group $H$.

In 2013, Omer et al. [10] investigated the relation between the graph of conjugacy classes with the probability of metacyclic 2-group fixes a set. Meanwhile in the same year, Moradipour et al. [11] studied the relation between non-commuting graphs with some metacyclic 2 groups.

In 2016, Sarmin et al. [12] explored the relation between the probability for dihedral group of order $2 n$ and graphs including generalized conjugacy class graph and orbit graph. In 2010, Darafsheh et al. [13] studied the characteristics of non-commuting graph of a finite group including the number of edges and the chromatic number in general.

\section{PRELIMINARIES}

In this section, some basic concepts in group theory and graph theory that will be used in obtaining the results are provided.

Definition 1 [14] $p$-Group

A $p$-group is defined as the group that has its order as prime power, labelled as $p^{n}$, where $p$ is prime and $n$ is non-negative integers.

Definition 2 [14] Conjugate of an Element

If $G$ is a group and $a \in G$, then a conjugate of $a$ is any element in $G$ of the form gag $^{-1}$ where $g \in G$.

Definition 3 [15] Conjugacy Class of an Element

Let $a, b \in G$. We say $a$ and $b$ are conjugate in $G$ (and call $b$ a conjugate of $a$ ) if $x^{-1} a x=b$ for some $x$ in $G$. The conjugacy class of $a$ is the set $\operatorname{cl}(a)=\left\{x^{-1} a x \mid x \in G\right\}$.

Definition 4 [16] Graph

A pair of the set vertices, $V$ and the set of edges, $E$, labelled as $\Gamma=$ $(V, E)$. The elements of $V$ are the vertices of $\Gamma$ and the elements of $E$ are the lines that combine two elements of $V$.

Definition 5 [17] Complete Graph

A complete graph is a simple graph in which every pairs of distinct vertices are adjacent. The complete graph with $n$ vertices denoted as $K_{n}$.

\section{Definition 6 [4] Conjugate Graph}

A graph is called a conjugate graph, denoted as $\Gamma_{G}^{c o n j}$, if it is associated to a non-abelian group $G$ with vertex set $G / Z(G)$ such that two distinct vertices are joined by an edge if they are conjugate. 
The classification of groups of order $p^{4}$ is given by Burnside in [18]. The non-abelian group of order $p^{4}$ that will be focused in this paper is given in the following:

$$
G=\left\langle x, y \mid x^{p^{2}}=y^{p^{2}}=1, x^{y}=x^{p+1}\right\rangle .
$$

\section{RESULTS AND DISCUSSION}

This section provides the results of the conjugate graphs for the nonabelian group $G$ of order $p^{4}$ for $p=2$ and 3 given as in the following theorems.

Theorem 1 Let $G=\left\langle x, y \mid x^{p^{2}}=y^{p^{2}}=1, x^{y}=x^{p+1}\right\rangle$ be a nonabelian $p$-group of order $p^{4}$ where $p=2$. Then, the conjugate graph of $G, \Gamma_{G}^{c o n j}$ is a union of six complete graphs with two vertices.

Proof: Suppose $p=2$, then $G=\left\langle x, y \mid x^{4}=y^{4}=1, x^{y}=x^{3}\right\rangle$. Thus the elements of $G$ is listed as follows:

$G=\left\{1, x^{2}, x^{2} y^{2}, y^{2}, x, x^{3}, y, x^{2} y, x y, x^{3} y, x y^{2}, x^{3} y^{2}, y^{3}, x^{2} y^{3}, x y^{3}, x^{3} y^{3}\right\}$.

By using Definition 3, the conjugacy classes of $G$ are listed in the following:

$$
\begin{array}{cl}
\text { i. } & \operatorname{cl}(1)=\{1\}, \\
\text { ii. } & \operatorname{cl}\left(x^{2}\right)=\left\{x^{2}\right\}, \\
\text { iii. } & \operatorname{cl}\left(x^{2} y^{2}\right)=\left\{x^{2} y^{2}\right\}, \\
\text { iv. } & \operatorname{cl}\left(y^{2}\right)=\left\{y^{2}\right\}, \\
\text { v. } & \operatorname{cl}(x)=\left\{x, x^{3}\right\}=\operatorname{cl}\left(x^{3}\right), \\
\text { vi. } & \operatorname{cl}(y)=\left\{y, x^{2} y\right\}=\operatorname{cl}\left(x^{2} y\right), \\
\text { vii. } & \operatorname{cl}(x y)=\left\{x y, x^{3} y\right\}=\operatorname{cl}\left(x^{3} y\right), \\
\text { viii. } & \operatorname{cl}\left(x y^{2}\right)=\left\{x y^{2}, x^{3} y^{2}\right\}=\operatorname{cl}\left(x^{3} y^{2}\right), \\
\text { ix. } & \operatorname{cl}\left(y^{3}\right)=\left\{y^{3}, x^{2} y^{3}\right\}=\operatorname{cl}\left(x^{2} y^{3}\right), \\
\text { x. } & \operatorname{cl}\left(x y^{3}\right)=\left\{x y^{3}, x^{3} y^{3}\right\}=\operatorname{cl}\left(x^{3} y^{3}\right) .
\end{array}
$$

From the above, it can be seen that the center of the group $G, Z(G)=$ $\left\{1, x^{2}, y^{2}, x^{2} y^{2}\right\}$. The order of the group $G$ is 16 and the order of $Z(G)$ is four. Thus by Definition 6, the vertices of the conjugate graph of the group $G$ is 12 . Each of the six conjugacy classes has size two, hence the conjugate graph of group $G$ is a union of six complete graphs of $K_{2}$, as presented in Figure 1.

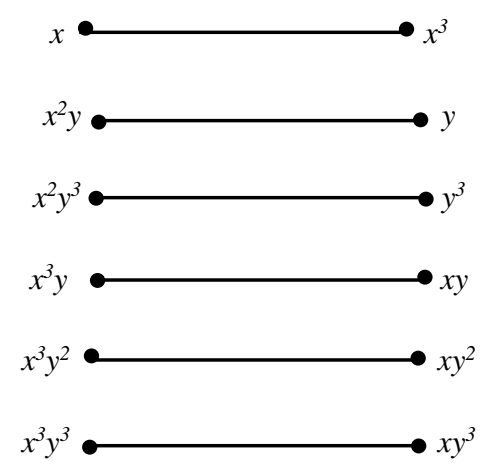

Fig. 1 The conjugate graph of the group $G$ with $p=2$.

Theorem 2 Let $G=\left\langle x, y \mid x^{p^{2}}=y^{p^{2}}=1, x^{y}=x^{p+1}\right\rangle$ be a nonabelian $p$-group of order $p^{4}$ where $p=3$. Then, the conjugate graph of $G, \Gamma_{G}^{c o n j}$ is a union of 24 complete graphs with three vertices.
Proof: Suppose $p=3$, then $G=\left\langle x, y \mid x^{9}=y^{9}=1, x^{y}=x^{4}\right\rangle$. Thus the elements of $G$ is listed as follows :

$G=\left\{1, x^{3}, x^{6}, y^{3}, x^{3} y^{3}, x^{6} y^{3}, y^{6}, x^{3} y^{6}, x^{6} y^{6}, x, x^{4}, x^{7}, x^{2}, x^{5}, x^{8}, y, x^{3} y, x^{6} y\right.$, $x y, x^{4} y, x^{7} y, x^{2} y, x^{5} y, x^{8} y, y^{2}, x^{3} y^{2}, x^{6} y^{2}, x y^{2}, x^{4} y^{2}, x^{7} y^{2}, x^{2} y^{2}, x^{5} y^{2}, x^{8} y^{2}$, $x y^{3}, x^{4} y^{3}, x^{7} y^{3}, x^{2} y^{3}, x^{5} y^{3}, x^{8} y^{3}, y^{4}, x^{3} y^{4}, x^{6} y^{4}, x y^{4}, x^{4} y^{4}, x^{7} y^{4}, x^{2} y^{4}, x^{5} y^{4}$, $x^{8} y^{4}, y^{5}, x^{3} y^{5}, x^{6} y^{5}, x y^{5}, x^{4} y^{5}, x^{7} y^{5}, x^{2} y^{5}, x^{5} y^{5}, x^{8} y^{5}, x y^{6}, x^{4} y^{6}, x^{7} y^{6}, x^{2} y^{6}$, $x^{5} y^{6}, x^{8} y^{6}, y^{7}, x^{3} y^{7}, x^{6} y^{7}, x y^{7}, x^{4} y^{7}, x^{7} y^{7}, x^{2} y^{7}, x^{5} y^{7}, x^{8} y^{7}, y^{8}, x^{3} y^{8}, x^{6} y^{8}, x y^{8}$, $\left.x^{4} y^{8}, x^{7} y^{8}, x^{2} y^{8}, x^{5} y^{8}, x^{8} y^{8}\right\}$.

By using Definition 3, the conjugacy classes of $G$ are listed in the following:

$$
\begin{aligned}
& \text { i. } \quad \operatorname{cl}(1)=\{1\}, \\
& \text { ii. } \quad \operatorname{cl}\left(x^{3}\right)=\left\{x^{3}\right\}, \\
& \text { iii. } \quad c l\left(x^{6}\right)=\left\{x^{6}\right\} \text {, } \\
& \text { iv. } \quad c l\left(y^{3}\right)=\left\{y^{3}\right\} \text {, } \\
& \text { v. } \quad \operatorname{cl}\left(x^{3} y^{3}\right)=\left\{x^{3} y^{3}\right\}, \\
& \text { vi. } \quad \operatorname{cl}\left(x^{6} y^{3}\right)=\left\{x^{6} y^{3}\right\} \text {, } \\
& \text { vii. } \quad c l\left(y^{6}\right)=\left\{y^{6}\right\} \text {, } \\
& \text { viii. } \quad \operatorname{cl}\left(x^{3} y^{6}\right)=\left\{x^{3} y^{6}\right\} \text {, } \\
& \text { ix. } \quad c l\left(x^{6} y^{6}\right)=\left\{x^{6} y^{6}\right\} \text {, } \\
& \text { x. } \quad \operatorname{cl}(x)=\operatorname{cl}\left(x^{4}\right)=\operatorname{cl}\left(x^{7}\right)=\left\{x, x^{4}, x^{7}\right\}, \\
& \text { xi. } \quad \operatorname{cl}\left(x^{2}\right)=\operatorname{cl}\left(x^{5}\right)=\operatorname{cl}\left(x^{8}\right)=\left\{x^{2}, x^{5}, x^{8}\right\}, \\
& \text { xii. } \quad \operatorname{cl}(y)=\operatorname{cl}\left(x^{3} y\right)=\operatorname{cl}\left(x^{6} y\right)=\left\{y, x^{3} y, x^{6} y\right\} \text {, } \\
& \text { xiii. } \quad c l(x y)=\operatorname{cl}\left(x^{4} y\right)=\operatorname{cl}\left(x^{7} y\right)=\left\{x y, x^{4} y, x^{7} y\right\} \text {, } \\
& \text { xiv. } \quad \operatorname{cl}\left(x^{2} y\right)=\operatorname{cl}\left(x^{5} y\right)=\operatorname{cl}\left(x^{8} y\right)=\left\{x^{2} y, x^{5} y, x^{8} y\right\} \text {, } \\
& \text { xv. } \quad \operatorname{cl}\left(y^{2}\right)=\operatorname{cl}\left(x^{3} y^{2}\right)=\operatorname{cl}\left(x^{6} y^{2}\right)=\left\{y^{2}, x^{3} y^{2}, x^{6} y^{2}\right\} \text {, } \\
& \text { xvi. } \quad \operatorname{cl}\left(x y^{2}\right)=\operatorname{cl}\left(x^{4} y^{2}\right)=\operatorname{cl}\left(x^{7} y^{2}\right)=\left\{x y^{2}, x^{4} y^{2}, x^{7} y^{2}\right\} \text {, } \\
& \text { xvii. } \quad \operatorname{cl}\left(x^{2} y^{2}\right)=\operatorname{cl}\left(x^{5} y^{2}\right)=\operatorname{cl}\left(x^{8} y^{2}\right)=\left\{x^{2} y^{2}, x^{5} y^{2}, x^{8} y^{2}\right\} \text {, } \\
& \text { xviii. } \quad \operatorname{cl}\left(x y^{3}\right)=\operatorname{cl}\left(x^{4} y^{3}\right)=\operatorname{cl}\left(x^{7} y^{3}\right)=\left\{x y^{3}, x^{4} y^{3}, x^{7} y^{3}\right\} \text {, } \\
& \text { xix. } \quad \operatorname{cl}\left(x^{2} y^{3}\right)=\operatorname{cl}\left(x^{5} x^{3}\right)=\operatorname{cl}\left(x^{8} y^{3}\right)=\left\{x^{2} y^{3}, x^{5} y^{3}, x^{8} y^{3}\right\}, \\
& \text { xx. } \quad \operatorname{cl}\left(y^{4}\right)=\operatorname{cl}\left(x^{3} y^{4}\right)=\operatorname{cl}\left(x^{6} y^{4}\right)=\left\{y^{4}, x^{3} y^{4}, x^{6} y^{4}\right\} \text {, } \\
& \text { xxi. } \quad \operatorname{cl}\left(x y^{4}\right)=\operatorname{cl}\left(x^{4} y^{4}\right)=\operatorname{cl}\left(x^{7} y^{4}\right)=\left\{x y^{4}, x^{4} y^{4}, x^{7} y^{4}\right\} \text {, } \\
& \text { xxii. } \quad \operatorname{cl}\left(x^{2} y^{4}\right)=\operatorname{cl}\left(x^{5} y^{4}\right)=\operatorname{cl}\left(x^{8} y^{4}\right)=\left\{x^{2} y^{4}, x^{5} y^{4}, x^{8} y^{4}\right\} \text {, } \\
& \text { xxiii. } \quad \operatorname{cl}\left(y^{5}\right)=\operatorname{cl}\left(x^{3} y^{5}\right)=\operatorname{cl}\left(x^{6} y^{5}\right)=\left\{y^{5}, x^{3} y^{5}, x^{6} y^{5}\right\} \text {, } \\
& \text { xxiv. } \quad \operatorname{cl}\left(x y^{5}\right)=\operatorname{cl}\left(x^{4} y^{5}\right)=\operatorname{cl}\left(x^{7} y^{5}\right)=\left\{x y^{5}, x^{4} y^{5}, x^{7} y^{5}\right\} \text {, } \\
& \text { xxv. } \quad \operatorname{cl}\left(x^{2} y^{5}\right)=\operatorname{cl}\left(x^{5} y^{5}\right)=\operatorname{cl}\left(x^{8} y^{5}\right)=\left\{x^{2} y^{5}, x^{5} y^{5}, x^{8} y^{5}\right\} \text {, } \\
& \text { xxvi. } \quad \operatorname{cl}\left(x y^{6}\right)=\operatorname{cl}\left(x^{4} y^{6}\right)=\operatorname{cl}\left(x^{7} y^{6}\right)=\left\{x y^{6}, x^{4} y^{6}, x^{7} y^{6}\right\} \text {, } \\
& \text { xxvii. } \quad \operatorname{cl}\left(x^{2} y^{6}\right)=\operatorname{cl}\left(x^{5} y^{6}\right)=\operatorname{cl}\left(x^{8} y^{6}\right)=\left\{x^{2} y^{6}, x^{5} y^{6}, x^{8} y^{6}\right\}, \\
& \text { xxviii. } \quad \operatorname{cl}\left(y^{7}\right)=\operatorname{cl}\left(x^{3} y^{7}\right)=\operatorname{cl}\left(x^{6} y^{7}\right)=\left\{y^{7}, x^{3} y^{7}, x^{6} y^{7}\right\} \text {, } \\
& \text { xxix. } \quad \operatorname{cl}\left(x y^{7}\right)=\operatorname{cl}\left(x^{4} y^{7}\right)=\operatorname{cl}\left(x^{7} y^{7}\right)=\left\{x y^{7}, x^{4} y^{7}, x^{7} y^{7}\right\} \text {, } \\
& \text { xxx. } \quad \operatorname{cl}\left(x^{2} y^{7}\right)=\operatorname{cl}\left(x^{5} y^{7}\right)=\operatorname{cl}\left(x^{8} y^{7}\right)=\left\{x^{2} y^{7}, x^{5} y^{7}, x^{8} y^{7}\right\}, \\
& \text { xxxi. } \quad \operatorname{cl}\left(y^{8}\right)=\operatorname{cl}\left(x^{3} y^{8}\right)=\operatorname{cl}\left(x^{6} y^{8}\right)=\left\{y^{8}, x^{3} y^{8}, x^{6} y^{8}\right\}, \\
& \text { xxxii. } \quad \operatorname{cl}\left(x y^{8}\right)=\operatorname{cl}\left(x^{4} y^{8}\right)=\operatorname{cl}\left(x^{7} y^{8}\right)=\left\{x y^{8}, x^{4} y^{8}, x^{7} y^{8}\right\}, \\
& \text { xxxiii. } \quad \operatorname{cl}\left(x^{2} y^{8}\right)=\operatorname{cl}\left(x^{5} y^{8}\right)=\operatorname{cl}\left(x^{8} y^{8}\right)=\left\{x^{2} y^{8}, x^{5} y^{8}, x^{8} y^{8}\right\} \text {. }
\end{aligned}
$$

From the above, it can be seen that the center of the group $G, Z(G)$ $=\left\{1, x^{3}, x^{6}, y^{3}, y^{6}, x^{3} y^{3}, x^{6} y^{3}, x^{3} y^{6} x^{6} y^{6}\right\}$. The order of the group $G$ is 81 and the order of $Z(G)$ is nine. Thus by Definition 6 , the vertices of the conjugate graph of the group $G$ is 72 . Each of the 24 conjugacy class has size three, hence the conjugate graph of group $G$ is a union of 24 complete graphs of $K_{3}$, as presented in Figure 2. 

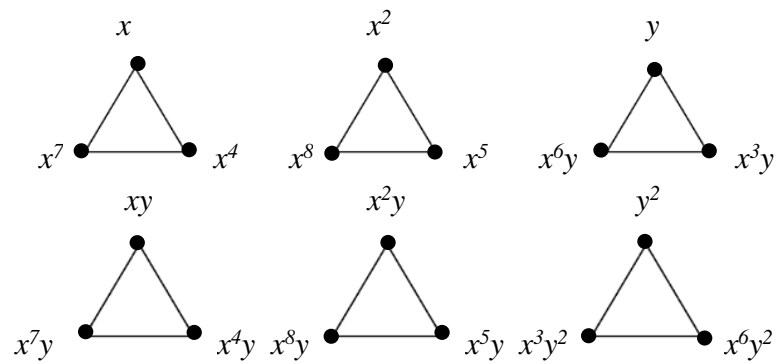
$x^{2} y$

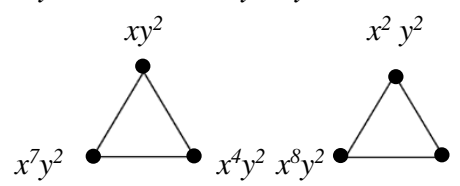

$x^{2} y^{2}$

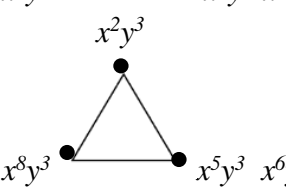

$y^{4}$
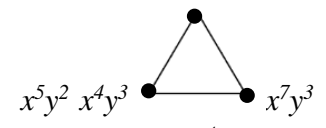

$x y^{3}$
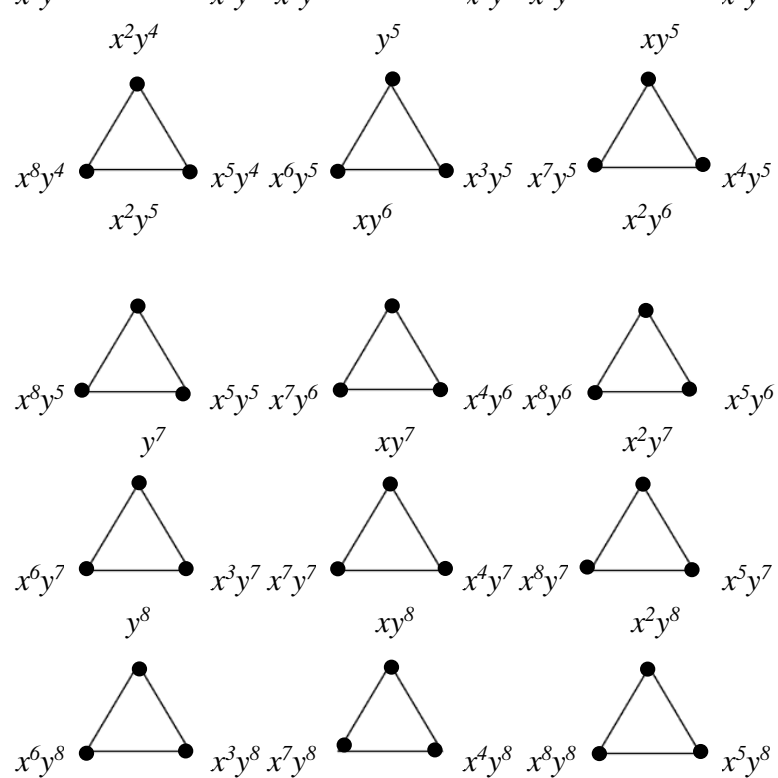

Fig. 2 The conjugate graph of the group $G$ with $p=3$.

\section{CONCLUSION}

As a conclusion, the conjugate graphs for the non-abelian groups of order $p^{4}$ turned out to be the union of six complete graphs with two vertices and the union of 24 complete graphs with three vertices for $p$ $=2$ and $p=3$ respectively.

\section{ACKNOWLEDGEMENT}

This work was financially supported by Ministry of Higher Education Malaysia and Universiti Teknologi Malaysia under the Research University Grant Scheme (GUP) for vote no. 11J96 and $13 \mathrm{H} 79$.

\section{REFERENCES}

[1] Laffey, T. J. 1978. Centralizers of elementary abelian subgroups in finite p-groups. Journal of Algebra. 51(1), 88-96.

[2] Zainal, R., Mohd Ali, N. M., Sarmin, N. H., \& Rashid, S. 2013. On the non-abelian tensor square of groups of order $p^{4}$ where $p$ is an odd prime. Science Asia 39S, 16-8.

[3] Rashid, S., Nawi, A. A., Zainal, R., Mohd Ali, N. M., \& Sarmin, N. H. 2014. The use of Groups, Algorithms and Programming (GAP) software in calculating the commutator subgroup and centre of groups of order $p^{3} q$. Science Asia. 40S, 73-77.

[4] Erfanian, A., Tolue, B. 2012. Conjugate graphs of finite groups. Discrete Mathematics, Algorithms and Applications. 4(2), 1250035.

[5] Bertram, E. A., Herzog, M., \& Mann, A. 1990. On a graph related to conjugacy classes of groups. Bulletin of the London Mathematical Society. 22(6), 569-575.

[6] Segev, Y. 2001. The commuting graph of minimal nonsolvable groups. Geometriae Dedicata. 88(1), 55-66.

[7] Akbari, S., Mohammadian, A., Radjavi, H., Raja, P. 2006. On the diameters of commuting graphs. Linear Algebra and its Applications. 418(1), 161-176.

[8] Bundy, D. 2006. The connectivity of commuting graphs. Journal of Combinatorial Theory, Series A. 113(6), 995-1007.

[9] Darafsheh, M. R. 2009. Groups with the same non-commuting graph. Discrete applied mathematics. 157(4), 833-837.

[10] Omer, S. M. S., Moradipour, K., Erfanian, A. 2013. The Probability That an Element of a Group Fixes a Set and Its Graph Related to Conjugacy Classes. Journal of Basic and Applied Scientific Research. 3(10), 369-380.

[11] Moradipour, K., Sarmin, N. H., Erfanian, A. 2013. On Non-commuting Graphs of Some Finite Groups. International Journal of Applied Mathematics and Statistics. 45(15), 473-476.

[12] Sarmin, N. H., El-sanfaz, M. A., Omer, S. M. S. 2016. Groups and graphs in probability theory. In AIP Conference Proceedings. 1750: 50012.

[13] Darafsheh, M. R., Bigdely, H., Bahrami, A., Monfared, M. D. 2010. Some results on non-commuting graph of a finite group. Italian Journal of Pure and Applied Mathematics. 27, 107-118.

[14] Rotman, J. J. 2002. Advanced Modern Algebra. USA: Pearson Education.

[15] Xiong, B. and Zheng, Z. 2010. Graph Theory. Shanghai, China: East China Normal University Press.

[16] Diestel, R. 2005. Graph Theory. ( ${ }^{\text {rd }}$ Edition). Germany: Springer.

[17] Wilson, R. J. 1972. Introduction to Graph Theory. USA: Academic Press.

[18] Burnside,W. 1911. Theory of groups of finite order. ( $2^{\text {nd }}$ Edition). Cambridge: Cambridge University Press 